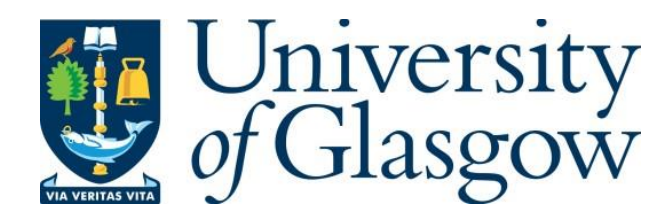

Baysan, U. (2017) Lawful mimickers. Analysis, 77(3), pp. 488-494.

(doi:10.1093/analys/anx086)

This is the author's final accepted version.

There may be differences between this version and the published version.

You are advised to consult the publisher's version if you wish to cite from it.

http://eprints.gla.ac.uk/141775/

Deposited on: 22 June 2017

Enlighten - Research publications by members of the University of Glasgow http://eprints.gla.ac.uk 


\title{
LAWFUL MIMICKERS
}

\section{Umut Baysan}

\begin{abstract}
The nomic view of dispositions holds that properties confer dispositions on their bearers with nomological necessity. The argument against nomic dispositions challenges the nomic view: if the nomic view is true, then objects don't have dispositions, but 'mimic' them. This paper presents an explication of disposition conferral which shows that the nomic view is not vulnerable to this objection.
\end{abstract}

Keywords: Dispositions; Laws of nature; Mimicking; The Nomic View

\section{Introduction}

The nomic view of dispositions holds that properties confer dispositions on their bearers with nomological necessity, where nomological necessity is understood as necessity dictated by the laws of nature. Recent work on dispositions and laws of nature has challenged the plausibility of the nomic view. Alongside the familiar objections to such a view that it posits a redundant role (i.e., governing of properties and dispositions) and an inappropriate filler for that role (i.e., the laws of nature $)^{1}$, there is now a new and worrying objection that dispositions that are conferred on objects with the help of the laws of nature can't be dispositions; in other words, if the nomic view is true, then properties don't confer dispositions. ${ }^{2}$ This paper presents an explication of disposition conferral which shows that the nomic view is not vulnerable to this latter type of objection.

\section{The argument against nomic dispositions}

If the nomic view is correct, then what dispositions a property confers on its bearers partly depends on the laws of nature, and properties can confer dispositions on their bearers even though they are not essentially dispositional properties. However, an argument recently developed by Contessa (2015) suggests that the nomic view can't explain disposition conferral. Call this 'the argument against nomic dispositions':

(i) Any disposition is either supervenient on the intrinsic properties of the object that has it, or it is not; call the supervenient ones 'intrinsic dispositions' and the rest 'extrinsic dispositions'. 3

(ii) If the nomic view is true, then no disposition that is conferred by a property is an intrinsic disposition.

\footnotetext{
${ }^{1}$ See Cartwright (1983) and Mumford (2004) for such objections.

${ }^{2}$ See Contessa (2015) for this latter type of objection.

${ }^{3}$ Contessa (2015) understands intrinsicality of a disposition in terms of supervenience on intrinsic properties, and extrinsicality of a disposition in terms of the failure of such supervenience (ibid: 163). Whether this is the right way to understand the intrinsicality (or extrinsicality) of a disposition is not relevant to the success of the argument, as what matters is actually the distinction between supervenient and non-supervenient dispositions. For further discussion of intrinsic and extrinsic dispositions, see McKitrick (2003), Heil (2003), and Yates (2016).
} 
(iii) If the nomic view is true, then no disposition that is conferred by a property is an extrinsic disposition.

(iv) If the nomic view is true, then no disposition that is conferred by a property is an intrinsic disposition or an extrinsic disposition.

(v) Therefore, if the nomic view is true, then no disposition is conferred.

But how properties confer dispositions on objects is what the nomic view is supposed to explain, so (v) is a highly problematic consequence for the nomic view. In what follows, I will argue that (iii) is false and hence the conclusion of this argument can be rejected. ${ }^{4}$

\section{Laws of nature and extrinsic dispositions}

What might be the reason to think that (iii) is true? Extrinsic dispositions are dispositions that don't supervene on the intrinsic properties of the objects that have them. So no intrinsic property (or combination thereof) will be sufficient for having a given extrinsic disposition. Extrinsic properties, some of which make reference to the laws of nature in that world, will have to be included in the supervenience base of a given extrinsic disposition. Contessa argues that if the nomic view is true, then extrinsic dispositions are not really dispositions, and hence properties don't confer extrinsic dispositions (ibid: 165-168). His argument appeals to the notion of mimicking:

(a) If the nomic view is true, then cases where objects seem to have extrinsic dispositions are cases of mimicking.

(b) Paradigmatically, cases of mimicking are cases where objects don't really have the dispositions they seem to have.

Therefore,

(iii) If the nomic view is true, then no disposition that is conferred by a property is an extrinsic disposition.

Why should we accept (a) and (b)? Let's start with (b). Cases of mimicking are typically given as counterexamples to what has come to be known as the 'simple conditional analysis' (SCA) of dispositions. According to the SCA, $\mathrm{x}$ is disposed to (manifest) $M$ when (stimulus condition) $S$ obtains if and only if $\mathrm{x}$ would $M$ if it were the case that $S$. A very simplified example is as follows: a vase is fragile if and only if it would break if it were struck. In the typical cases of mimicking, the right-hand side of the biconditional that is associated with a disposition is true (because external factors replicate the associated stimulus conditions and lead to the associated manifestation) whereas the left-hand side is false (because the object doesn't have the disposition in question). For example, a vase might be non-fragile, but a wizard might just cast a spell on it so that it breaks when struck despite not being fragile. ${ }^{5}$ Given that this is how

\footnotetext{
${ }^{4}$ Premise (i) is analytic; (iv) is a valid inference from the previous premises. Due to considerations of space, I will not discuss (ii), but for what it is worth, I think it is true insofar as the nomic view is committed to the contingency of laws of nature (see Armstrong 1997).

${ }^{5}$ See Lewis (1997) for similar examples. Mimics are not to be confused with 'finks' (e.g. Martin 1994). Fink cases constitute a separate class of apparent counterexamples to the SCA but they present other
} 
mimicking is paradigmatically understood and that (b) doesn't seem to say anything on top of what paradigmatic cases of mimicking are, we can accept (b).

What about (a)? What are the reasons for thinking that if the nomic view is true, then alleged cases of extrinsic dispositions are in fact cases of mimicking? Contessa motivates this point via a number of analogies (2015: 165-168), which I shall summarise as follows. We know that electrons don't have the disposition to annihilate protons in their vicinity. But suppose that a wizard casts a spell on an electron so that whenever that electron comes close to a proton, it annihilates the proton. So, from the point of view of an observer who doesn't know anything about the spell, this electron seems to have the disposition to annihilate nearby protons. This is clearly a case of mimicking: electrons don't have the disposition to annihilate nearby protons, but due to the spell that the wizard casts, whenever this electron gets close to a proton, the proton gets annihilated. Imagine now that our wizard casts the same spell on all electrons. Now every electron would seem to have the disposition to annihilate protons in its vicinity, but this is still a case of mimicking. Importantly, nothing would have changed if, instead of a spell, a law of nature made it the case that whenever an electron comes close enough to a proton, the proton gets annihilated. That is, suppose that it is somehow a contingent law of nature in the imagined scenario that electrons annihilate nearby protons. Contessa argues that if the previous scenarios describe cases of mimicking, then, by analogy, this latter scenario should be describing a case of mimicking too. If the laws of nature in this imagined world make electrons annihilate nearby protons, then they make electrons mimic the disposition to annihilate nearby protons, exactly as the wizard does. According to Contessa, this suggests that electrons in this imagined world don't genuinely have this disposition (2015: 166). Now, if the nomic view is true, what makes the actual cases of disposition conferral different from these imaginary cases, which are clearly cases of mimicking? Aren't the laws of nature still making it the case that certain regularities obtain in counterfactual-supporting ways? So, there seems to be a strong case for premise (a) too.

Having seen why cases of extrinsic dispositions are indistinguishable from cases of mimicking, let us go back to the argument for (iii). Fortunately for the defenders of the nomic view, even if we accept premises (a) and (b), the conclusion doesn't seem to follow. What we should get from (a) and (b) is instead the following conclusion:

(iii*) If the nomic view is true, then cases where objects seem to have extrinsic dispositions belong to a type $T$ such that the paradigmatic examples of $T$ are cases where objects don't really have the extrinsic dispositions they seem to have.

Although (iii*) may support (iii), it doesn't entail it. Paradigmatic examples of mimicking may indeed be cases where objects don't really have the relevant dispositions, nevertheless some examples of mimicking may be cases of real dispositions. For a simple argument for the claim that mimicking $\mathrm{x}$ doesn't entail not having/being $\mathrm{x}$, just think of a case where a hypochondriac mimics the symptoms of an illness and yet unknowingly has the illness in question.

difficulties. In some fink cases, as soon as the stimulus condition that is associated with a disposition $D$ holds, an object with $D$ ceases to have $D$, therefore despite having $D$ (at the moment of stimulation) and being subjected to the right stimulus condition, the manifestation doesn't follow. In other fink cases (sometimes called 'reverse-finks'), an object without $D$ acquires $D$ as soon as the associated stimulus condition obtains, thereby the associated manifestation takes place. See Bird (2007) for detailed discussion of these cases. 
I think the analogies we considered give us very strong reasons to think that the laws of nature, as the nomic view sees them, have a crucial role in making it the case that certain regularities obtain in counterfactual-supporting ways. Their role is at least as central as the role of the spells in some imaginary cases of mimicking. Does this mean that the nomic view implies that there are no extrinsic dispositions? I don't think so. One might find it problematic to give the laws of nature this sort of role in governing certain regularities, but this is part of the nomic view: a law of nature is not a redundant thing. After all, if the nomic view is true, unless the laws of nature are the way they are, regularities may not hold in counterfactual-supporting ways.

In the next section, I will show that the nomic view's understanding of disposition conferral might well be understood as mimicking, re-emphasising that mimicking doesn't entail the nonexistence of the disposition that is mimicked. So, I will argue that premise (a) can be true and that this is not a problem for the nomic view.

\section{Disposition conferral and mimicking}

In the previous section, I argued that the argument for (iii) at best illuminates how much the nomic view relies on the laws of nature, but that this shouldn't come as a surprise, as the defender of the nomic view can happily accept this. Now, I wish to show how mimicking can be incorporated into the nomic view's understanding of disposition conferral, implying that mimicking might as well be a natural and widespread phenomenon (as far as the nomic view is concerned).

In order for the case against the nomic view to work, the intended conclusion (IC) must be this:

(IC) If the nomic view is true, then no disposition is conferred.

I hope I have persuaded you that the analogies we considered in Section 3 at best support the following claim, which I shall call (RC) - for 'Revised Conclusion':

(RC) If the nomic view is true, then what seem to be cases of dispositions are indistinguishable from some cases of mimicking. ${ }^{6}$

Now, it is important for my defence of the nomic view that (RC) doesn't entail (IC). And here is the reason why it does not: it is perfectly possible - and in fact reasonable - for a proponent of the nomic view to understand disposition conferral (DC) in the following sense:

(DC) A property $P$ in a world $w$ confers on its bearers a disposition $D$ if and only if the laws of nature in $w$ make it the case that bearers of $P$ display the stimulus-manifestation patterns that are characteristic of $D .^{7}$

\footnotetext{
${ }^{6}$ In Section 3, we only considered extrinsic dispositions, but the point is meant to apply to all dispositions, as we can agree with Contessa that if the nomic view is true, all dispositions are extrinsic dispositions. See footnote 4.

${ }^{7}$ This is meant to apply to all bearers of $P$ in $w$ except where $D$ is finked or masked. Finks are explained in footnote 5 above. In mask cases, dispositions are not manifested despite the occurrence of the stimulus conditions. For example, a fragile vase covered by protective material might not break when it is struck. So a more precise version of (DC) should take into account such cases. This is a notoriously difficult issue, and it deserves a separate treatment. Thanks to an anonymous referee for pointing this out.
} 
As I said in the previous section, if one holds the nomic view, one will reasonably think that a law of nature has a central role in making it the case that certain counterfactual-supporting regularities obtain. And if (DC) is true, then (RC) doesn't entail (IC). This is because for something to mimic a disposition is for it to display the stimulus-manifestation pattern that is characteristic of a disposition, and I have argued that this is what the laws of nature enable things to do, if the nomic view is true. Therefore, (RC) doesn't entail (IC).

One might object as follows: it is not true that for something to mimic a disposition is for it to display the associated stimulus-manifestation pattern; after all, mimicking is introduced as a phenomenon whereby the disposition that is mimicked does not actually exist (in the object that appears to have it). But what I am challenging here is exactly this way of understanding mimicking. I think it is reasonable to think that mimicking a disposition $D$ doesn't imply that $D$ doesn't exist; instead, it leaves it open whether $D$ exists. In that sense, because of the possibility of mimicking, one might not infallibly know if something has a disposition it appears to have. Once again, think about our fragile vase. It is conceivable that the vase is fragile, but that it breaks not as a results of its fragility, but as a result of a spell which guarantees that it would break if dropped on a hard surface, thus pre-empting the usual manifestation of the vase's fragility. The spell effectively enables the vase to mimic fragility, as the causally operative condition for the vase's breaking is the spell, not its fragility. Nevertheless, we stipulated that the vase is fragile. Thus, mimicking doesn't entail the nonexistence of what is being mimicked. It is true that in cases where mimicking is used as a counterexample to the SCA, objects don't have the dispositions that they appear to have. But this needn't be the rule. And in fact, if I am right, then the defenders of the nomic view should happily endorse the prevalence of mimicking.

Before concluding, allow me to consider an alternative response to the argument against nomic dispositions, which can also be seen as an objection to my understanding of mimicking. According to this alternative response, Contessa's argument for (a) trades on the idea that cases of mimicking are supposed to be somehow exceptional. But if cases of mimicking are exceptional, then there is an immediate response to the series of analogies that Contessa presents to support (a). The case with the wizard and one electron is an exceptional case. The case with all electrons (in a world) and a wizard is an exceptional case too; despite the widespread influence of the wizard in that case, the wizard's interference with the behaviours of electrons is not normal and involves an element of exceptionality. But when it comes to the case with all electrons and the laws of nature (instead of the wizard), we don't have an element of exceptionality anymore. After all, if a counterfactual pattern is underwritten by some universal laws of nature (as the defenders of the nomic view hold) then we don't have a case of exceptionality in such a case. So, according to this alternative response, the last case is not a case of mimicking due to failing to be exceptional; hence (a) is false. ${ }^{8}$

This alternative response can be seen as an objection to my understanding of mimicking because, according to my construal, if the nomic view is true, then mimicking is a widespread and unexceptional phenomenon. But a good account of mimicking should accommodate its exceptionality. So, this alternative response seems to undermine my proposal.

Note that this objection can be rebutted if my understanding of mimicking can accommodate a distinction between ordinary and extraordinary cases. Ordinary cases of mimicking are cases

\footnotetext{
${ }^{8}$ Thanks to an anonymous referee for presenting this alternative response to the argument against nomic dispositions.
} 
where things go according to what we expect. Our expectations are justified in virtue of our credence in lawful connections between certain stimulus conditions and manifestations. In extraordinary cases however, objects display certain stimulus-manifestation patterns due to extraordinary circumstances (e.g., interferences by wizards). In such circumstances, ordinary courses of events are broken, so non-fragile things break when they are hit, electrons annihilate protons when they get near them, and so on. So, my understanding of mimicking has the resources to respond to this objection: interesting examples of mimicking have a degree of exceptionality due to the extraordinariness of the circumstances that lead to them.

\section{Conclusion}

I presented a model of disposition conferral that the defenders of the nomic view of dispositions can endorse. In so doing, I responded to the argument that if the nomic view is true, then extrinsic dispositions are not real dispositions, but are only mimicked. I argued that the nomic view can explain the conferral of extrinsic dispositions if the notion of mimicking is understood in an appropriate way. For something to mimic a disposition is for it to display the associated stimulus-manifestation pattern, and if the nomic view is true, laws of nature play an important role in bringing about such stimulus-manifestation pairings. ${ }^{9}$

\section{References}

Armstrong, D. M. 1997. A World of States of Affairs. Cambridge: Cambridge University Press. Bird, A. 2007. Nature's Metaphysics. Oxford: Oxford University Press.

Cartwright, N. 1983. How the Laws of Physics Lie. Oxford: Clarendon Press. Contessa, G. 2015. Only powers can confer dispositions. Philosophical Quarterly 65: 160176.

Heil, J. 2003. From an Ontological Point of View. Oxford: Oxford University Press.

Lewis, D. 1997. Finkish dispositions. Philosophical Quarterly 47: 143-158.

Martin, C. B. 1994. Dispositions and conditionals. Philosophical Quarterly 44: 1-8.

McKitrick, J. 2003. A case for extrinsic dispositions. Australasian Journal of Philosophy 81: 155-74.

Mumford, S. 2004 Laws in Nature. New York: Routledge.

Yates, D. 2016. Is powerful causation an internal relation? In The Metaphysics of Relations, ed. A. Marmodoro \& D. Yates, 138-156. Oxford: Oxford University Press.

\footnotetext{
${ }^{9}$ Many thanks to Katherine Baysan and Stephan Leuenberger for reading previous versions of the paper, and two anonymous referees of this journal for their very helpful comments. This publication was made possible through the support of a grant from the John Templeton Foundation. The opinions expressed in this publication are those of the author and do not necessarily reflect the views of the John Templeton Foundation.
} 